\title{
Sampling behavior of dairy cattle: Effects of variation in dietary energy density on behavior at the feed bunk
}

\author{
J. M. Huzzey, ${ }^{*}$ J. A. Fregonesi, ${ }^{*} \dagger$ M. A. G. von Keyserlingk, ${ }^{*}$ and D. M. Weary ${ }^{* 1}$ \\ *Animal Welfare Program, Faculty of Land and Food Systems, University of British Columbia, 2357 Main Mall, Vancouver, BC, V6T 1Z4, Canada \\ †Universidade Estadual de Londrina, Parana, CEP-86051-990, Brazil
}

\begin{abstract}
Factors affecting sampling behavior of cattle are poorly understood. The objectives of this study were to measure the effects of variation in feed quality on the feeding behavior of Holstein dairy heifers. Thirty-two heifers were housed in 4 groups of 8 . Each group pen had 8 distinct feeding stations. The total mixed ration (TMR) provided was low energy (TMR-L), moderate energy (TMR-M), or high energy (TMR-H). During trial 1 (d 1 to 8), heifers were offered a uniform baseline diet (TMR-M in all 8 feeding stations) interspaced with 2 uniform test diets on d 3 and 6 (TMR-L or TMR-H in all 8 feeding stations). During trial 2 (d 9 to 17) heifers were offered a nonuniform baseline diet ( 7 feeding stations with TMR-L and 1 feeding station with TMR-H) interspaced with 3 uniform test diets on d 11, 14, and 17 (TMR-L, TMR-M, or TMR-H in all 8 feeding stations). Heifers were observed in pairs $(\mathrm{n}=16)$ for $15 \mathrm{~min}$ following delivery of fresh feed. Relative to the uniform baseline period of trial 1, 31\% fewer switches occurred between feeding stations when offered TMR-H and $51 \%$ more switches when offered TMR-L. Relative to the nonuniform baseline of trial 2, $49 \%$ fewer, $27 \%$ fewer, and $25 \%$ more switches occurred during the TMR-H, TMR-M, and TMR-L treatments, respectively. In general, when heifers were offered a diet that was lower in energy density than that previously experienced, they spent less time at each feeding station and when offered a higher energy diet, heifers spent more time at each feeding station. The greater the contrast in energy density between the test and baseline diets, the greater the change in the behavioral response. Competitive interactions at the feed bunk were most frequent when TMR quality varied among the 8 feeding stations; during the nonuniform baseline period of trial 2, the number of competitive interactions was over 3.5 times higher than during all uniform dietary treatments. In summary, dairy heifers sample feed quality by changing feeding
\end{abstract}

Received June 1, 2012

Accepted September 6, 2012.

${ }^{1}$ Corresponding author: danweary@mail.ubc.ca locations at the feed bunk and this sampling behavior is affected by variation in diet quality along the feed bunk and across days.

Key words: heifer, diet quality, agonistic interaction, feeding behavior

\section{INTRODUCTION}

In nature and pasture-based management systems, the foraging decisions of cattle occur at several levels, including (1) when to feed, (2) where to feed, (3) what to consume, and (4) how to consume it. These decisions are based on abiotic factors, such as land topography, distance from water, and environmental conditions and biotic factors such as plant composition, quality, quantity, and morphology. Intake rate and postingestive feedback can then be used to integrate information obtained through diet selection to evaluate the suitability of a particular feeding site (Provenza, 1995). Other factors such as the social environment, degree of predation risk, health status, or dominant physiological state (e.g., lactating vs. nonlactating and pregnant) can also influence how and what cattle eat; for example, with an increased threat of predation animals may decrease time spent at a feeding site, restrict movements at a feeding site, or increase vigilance behavior (Lima, 1998).

The factors influencing the feeding decisions of cattle reared indoors have received far less attention; researchers are only now beginning to recognize some of the factors that influence feeding behavior of modern dairy cows. For example, research has shown that cows are highly motivated to eat during the hours following the delivery of fresh feed (DeVries and von Keyserlingk, 2005). Dairy cattle can also be selective in their dietary choices if given the opportunity. Leonardi and Armentano (2003) found cows preferentially sort for the grain component of a TMR, avoiding the longer forage components. Cows will also eat faster in competitive feeding environments such as those encountered during periods of overstocking (Olofsson, 1999; Proudfoot et al., 2009). These findings relate to feeding decisions involving when, what, and how dairy cattle eat at a feed 
bunk; however, no work has studied factors that may influence where cattle chose to eat along the length of the feed bunk.

Most intensive dairy farms feed cattle a TMR that is intended to be well mixed; the assumption is that all cattle will have access to a diet of uniform quality. Several factors, however, may introduce unexpected variation in diet quality both in space (i.e., along the length of a feed bunk) and in time (i.e., within and among days). These factors could be management related such as improper mixing or chopping of the TMR and not placing feed along the entire length of the feed bunk or be cow-level factors such as sorting behavior. Sampling behavior allows animals to locate feeding sites of higher quality (Stephens et al., 2007), but to sample animals must move between feeding locations, which increases time that the animal is not feeding. Abrupt changes in dietary energy density may increase short-term sampling frequency to the point that time available for feeding is compromised. The objective of this study was to measure the effects of unexpected variation in the energy density of the diet on sampling behavior of indoor-housed cattle.

\section{MATERIALS AND METHODS}

\section{Animals and Housing}

This study was conducted at the University of British Columbia's Dairy Education and Research Center (Agassiz, BC, Canada) from November to December 2009. All animals were cared for according to the guidelines of the Canadian Council on Animal Care (CCAC,
2009). Thirty-two Holstein dairy heifers were enrolled in this study. The average \pm standard deviation (range) BW and age of the experimental animals was 232.6 $\pm 55.1 \mathrm{~kg}(152-330 \mathrm{~kg})$, and $207.1 \pm 45.3 \mathrm{~d}(135-271$ d), respectively. The experimental animals were further divided into 4 groups of 8 heifers; groups were balanced for BW and age.

In sets of 2 (experimental design was replicated over time), groups were randomly assigned to 1 of 2 identical experimental freestall pens. Each pen (Figure 1) consisted of 13 deep-bedded sand stalls arranged in a 3-row formation. The feed barrier consisted of 15 headlock feeding stations. Along this feed barrier every second headlock was blocked to prevent access to the feed; this allowed for a total of 8 functional feeding stations within each experimental pen, each separated by $0.8 \mathrm{~m}$ (from center to center of nearest available feeding station). Feed bins (high-density polyethylene box measuring: $0.45 \times 0.35 \times 0.40 \mathrm{~m}$ in length, width and height) were positioned in front of each feeding station and secured to the headlock partitions with a chain. This arrangement of feed bins prevented a heifer at one feeding station from accessing feed from a neighboring feeding station; heifers had to physically move to a new feeding station to sample the feed at that location.

\section{Experimental Design}

This study was conducted as 2 trials that occurred in immediate succession. Groups were formed at least 7 $\mathrm{d}$ before the beginning of the first trial so that heifers could acclimate to grouping. Both trials were conducted over a period of $17 \mathrm{~d}$, with the first trial occurring

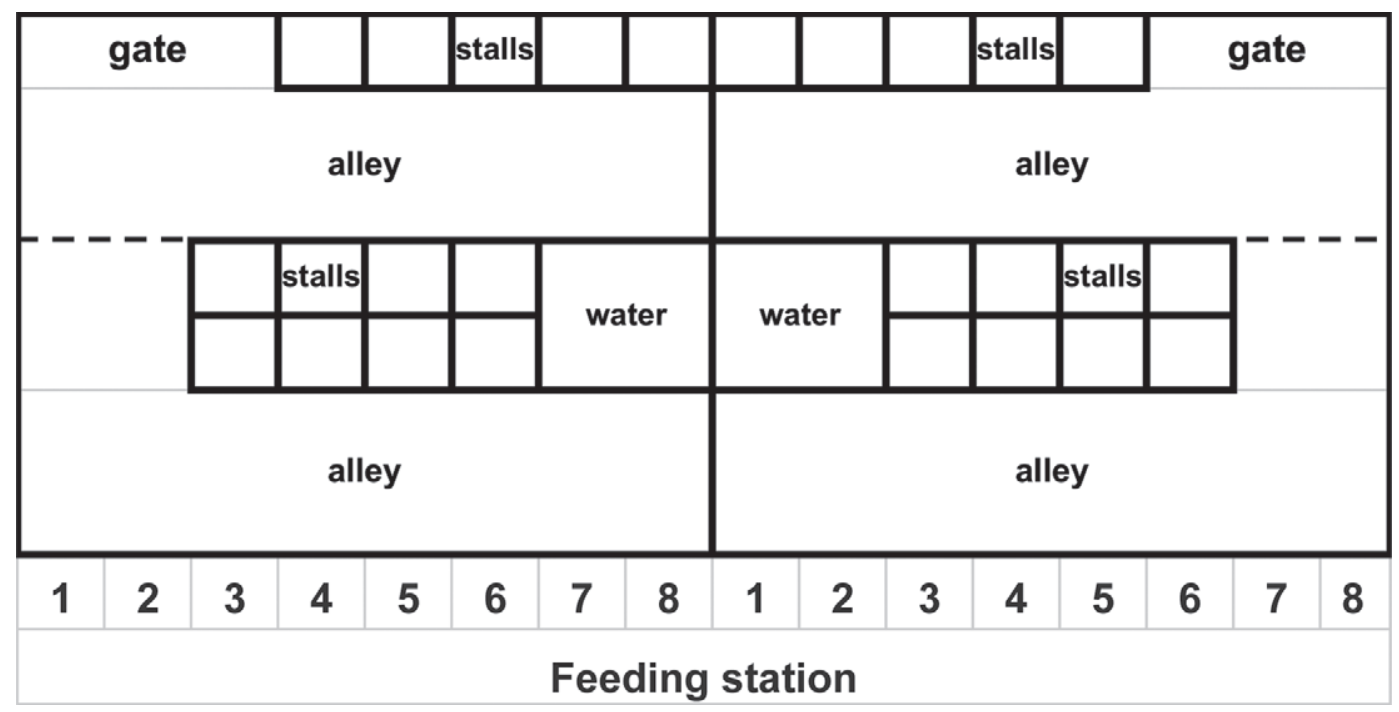

Figure 1. Experimental pen layout. Dashed line represents a movable gate. 
Table 1. Layout of experimental design

\begin{tabular}{lcll}
\hline Trial & Day & Baseline $^{1} /$ test & Diet offered $^{2}$ \\
\hline Trial 1 & 1 & Uniform & TMR-M \\
& 2 & Uniform & TMR-M \\
& 3 & Test 1 & TMR-L or TMR-H \\
& 4 & Uniform & TMR-M \\
& 5 & Uniform & TMR-M \\
& 6 & Test 2 & TMR-L or TMR-H \\
& 7 & Uniform & TMR-M \\
Trial 2 & 8 & Uniform & TMR-M \\
& 9 & Nonuniform & TMR-L +1 TMR-H \\
& 10 & Nonuniform & TMR-L or TMR-M or TMR-H \\
& 11 & Test 3 & TMR-L +1 TMR-H \\
& 12 & Nonuniform & TMR-L +1 TMR-H \\
& 13 & Nonuniform & TMR-L or TMR-M or TMR-H \\
& 14 & Test 4 & TMR-L +1 TMR-H \\
& 15 & Nonuniform & TMR-L +1 TMR-H \\
& 16 & Nonuniform & TMR-L or TMR-M or TMR-H \\
\hline
\end{tabular}

${ }^{1} \mathrm{~A}$ uniform baseline diet was fed between the test days during trial 1 [i.e., moderate-energy TMR (TMR-M) from all 8 feeding stations $]$. A nonuniform baseline diet was fed between test days during trial $2[7$ feeding stations with low-energy TMR (TMR-L) and 1 station with high-energy TMR (TMR-H)].

${ }^{2}$ On test days, groups were randomly assigned only 1 of the indicated diet types and on the following test day were assigned a different diet, such that by the end of the trial, each group received each of the test diets.

from $\mathrm{d} 1$ to 8 and the second from d 9 to 17 (Table 1 ). By manipulating the percentage of grain concentrate mixed into a base TMR, 3 treatments that differed in energy density were created: low energy (TMR-L), moderate energy (TMR-M), and high energy (TMR$\mathbf{H}$; Table 2). The ingredient composition of the grain concentrate mix is provided in Table 3 (DM: $89 \%$; CP: $16.9 \%$ of DM; ADF: $9.0 \%$ of DM; NDF: $24.4 \%$ of DM; and TDN: $79.4 \%$ of DM).

Heifers were fed for ad libitum intake, with fresh feed delivered daily at $0900 \mathrm{~h}$ after the previous day's orts were removed. Heifers had ad libitum access to water and a mineral salt lick for the duration of the study.

Trial 1 (Uniform Baseline). Heifers were fed TMR-M from all 8 feed bins during d 1 to 2, 4 to 5 , and 7 to 8 . On d 3 and 6 , heifers were fed either TMR-L or TMR-H, such that by the end of the trial each heifer received each diet.

Trial 2 (Nonuniform Baseline). Heifers were fed TMR-L from 7 of the feed bins and TMR-H from the eighth bin, with location of the TMR-H bin randomized across days. This nonuniform baseline was provided on d 9 to10, 12 to 13, and 15 to 16 . On d 11, 14, and 17 groups were pseudorandomly assigned to TMR-L, TMR-M, or TMR-H from all 8 bins, such that by the end of the trial each heifer was tested with each diet.

Observation Period. Before the morning delivery of fresh feed, heifers were moved to the rear of the pen to prevent access to the feeding area. After feed delivery, heifers were allowed to enter the feeding area in pairs for 15 min during which they were observed continuously. The period following fresh feed delivery is when feeding activity peaks (DeVries and von Keyserlingk, 2005); therefore, it was anticipated that the greatest behavioral responses to changes in the TMR quality would occur during the period immediately following access to the morning fresh feed. Further, the decision was made to cap the observation time to 15 min to avoid having a long duration of time separating the observation period of the first pair in the group from the last pair in the group. After each pair were observed, they were returned to the rear of the pen, fresh

Table 2. Ingredient composition of the 3 TMR diets $^{1}$

\begin{tabular}{|c|c|c|c|}
\hline \multirow[b]{2}{*}{ Ingredient } & \multicolumn{3}{|c|}{ Diet $[\mathrm{kg}$ of DM/heifer (\% of total DM)] } \\
\hline & TMR-L & TMR-M & TMR-H \\
\hline Fescue hay & $3.4(68)$ & $3.4(51)$ & $3.4(42)$ \\
\hline Corn silage & $1.1(22)$ & $1.1(17)$ & $1.1(13)$ \\
\hline Grass silage & $0.5(10)$ & $0.5(8)$ & $0.5(6)$ \\
\hline Grain concentrate mix & $0(0)$ & $1.6(24)$ & $3.2(39)$ \\
\hline
\end{tabular}


Table 3. Ingredient composition of the heifer grain concentrate mix (overall $\mathrm{DM}=89 \%$ )

\begin{tabular}{lc}
\hline Ingredient & Amount (\% as fed) \\
\hline Barley, rolled & 40.0 \\
Barley, fine ground & 21.7 \\
Millrun pellets & 15.0 \\
Distillers corn & 10.0 \\
Canola meal & 4.47 \\
Limestone, fine & 2.53 \\
Soybean meal & 2.50 \\
Molasses & 2.00 \\
Mineral and vitamin mix & 1.80 \\
\hline
\end{tabular}

feed was redistributed along the feed bunk, and the next pair was brought into the feeding area; this procedure was repeated until each pair was tested. After testing, all heifers were allowed free access to the feed bunk for the remainder of the day; the diet available for the remainder of the day was the same quality as that provided during the morning testing period. Pairs remained constant throughout both the trials.

\section{Behavioral Data}

Behaviors were monitored using digital video (Genetec Omnicast Surveillance System; Genetec Inc., Quebec, ON). One camera (WV-BP334 Digital black and white CCD camera; Panasonic, Secaucus, NJ) was located directly above the feeding area of each experimental pen and positioned so that the entire feed bunk length as well as the entire feeding area was visible. Heifers were marked with unique alphanumeric symbols using hair dye. Behaviors were recorded during test days during trial 1 (d 3 an 6 ) and trial 2 (d 11, 14, and 17), for 2 of the $6 \mathrm{~d}$ that heifers were fed the uniform baseline TMR during trial 1 (d 2 and 5), and for 3 of the $6 \mathrm{~d}$ that heifers were fed the nonuniform baseline TMR during trial 2 (d 10, 13, and 16). Data were not collected on the day following test days (or on the first day of each trial) to minimize carryover effects; further, because baseline data was averaged across multiple days, if an influence of test day diet existed on baseline responses, it would not have been any one test diet in particular driving this response.

A heifer was classified as feeding when its head (ears inclusive) was visible beyond the top rail of the feed barrier on the feed alley side of the pen. Sampling behavior was assessed by measuring movements (switches) between feeding stations. A switch was recorded when a heifer withdrew its head from one feeding station, moved to another station and resumed feeding. Feeding station interval (FSI) was defined as the length of time a heifer was feeding at a single feeding station. A successful displacement was noted when a heifer's head (actor) came in contact with a heifer that was feeding (reactor), resulting in the reactor withdrawing her head from the feeding station. A displacement attempt was recorded if an actor's head pressed against any portion of a feeding heifer's body but the contact did not cause the feeding heifer to leave the feed bunk. The measures of displacements and attempts to displace had high interobserver reliability (correlation $\mathrm{R}^{2}=1$ and 0.97 , respectively) likely because only 2 cows were observed at a time, thus allowing for more accurate assessments of behavior.

An FSI ratio was calculated to describe the degree of asymmetry in FSI between heifers within a pair for each treatment. The FSI ratio was calculated by dividing the longest FSI of the pair by the shortest FSI of the pair; an FSI ratio of 1 indicates that both heifers had the same FSI.

\section{Statistical Analysis}

For the analysis of feeding station switches, competitive interactions (sum of displacements and attempts to displace), FSI, and FSI ratio, the experimental unit was the pair $(\mathrm{n}=16)$, with events from both heifers in a pair added together (with the exception of the FSI ratio) to generate an overall observation per pair per day. Data collected over multiple baseline TMR days were averaged to create 1 observation per pair per baseline type (uniform or nonuniform). Data for trials 1 and 2 were analyzed separately. Differences in responses between the baseline and test days were analyzed using contrast statements in the MIXED procedure of SAS (version 9.2, 2009; SAS Institute Inc., Cary, NC). Logarithmic transformation was used whenever needed to comply with the assumptions of normality. When transformation was needed, least squares means and 95\% confidence intervals were back transformed to obtain the geometric mean and confidence interval on the original scale.

\section{RESULTS}

\section{Observational Analysis of Sampling Behavior}

Figure 2 provides examples from 2 experimental pairs of the typical pattern of switching behavior between bins during one of the nonuniform baseline days and during the days the pairs were offered TMR-H and TMR-L during trial 2. This observational analysis of movement patterns between feed bins suggests that when spatial variation in feed quality exists, heifers prefer to feed from the highest quality bin; this feed station, once discovered, was occupied for most of the 15 min observation period and most of the competitive 
interactions at the feed bunk occurred at this location. These examples also highlight the differences in sampling behavior when cattle are offered a different quality TMR across days. When transitioned to a uniformly distributed high-quality TMR, heifers appeared to have longer FSI and switch less frequently between feed stations. When offered a low-quality TMR, heifers appeared to respond by increasing switching frequency and having shorter FSI. Figure 2 is meant to provide a visual reference for the quantitative measures of sampling behavior that are reported in the following sections.

\section{Trial 1}

Relative to the days heifers were fed the uniform baseline diet (TMR-M), 51\% more feed station switches occurred when heifers were fed TMR-L and $31 \%$ fewer switches when fed TMR-H $(P<0.001$ and $P=0.02$, respectively; Figure 3A).

The frequency of competitive interactions at the feed bunk (displacements and attempts to displace) did not differ between days fed the uniform (TMR-M) baseline diet and the days fed either TMR-L or TMR-H $(P=$ 0.42 and $P=0.17$, respectively; Figure 3B).

During the days heifers were fed the uniform baseline diet, the average FSI $( \pm \mathrm{SE})$ was $128 \pm 28 \mathrm{~s}$; this was shorter than the FSI when fed TMR-H $(276 \pm 28 \mathrm{~s} ; P$
$<0.001)$ but similar to the FSI when fed TMR-L (85 $\pm 30 \mathrm{~s} ; P=0.30)$. Heifers within pairs responded symmetrically across treatments as evidenced by similar FSI ratios during the baseline treatment and during TMR-H and TMR-L test days [median FSI ratio (95\% $\mathrm{CI})=1.6$ (1.3 to 2.1 ), 2.0 (1.6 to 2.5), and 1.5 (1.1 to 1.9) respectively; $P \geq 0.14$ ].

\section{Trial 2}

Relative to the days heifers were fed the nonuniform baseline diet, $25 \%$ more feeding station switches occurred when heifers were fed TMR-L $(P=0.04), 27 \%$ fewer switches when fed TMR-M $(P=0.02)$, and $49 \%$ fewer switches when fed TMR-H $(P<0.001$; Figure $4 \mathrm{~A})$.

The frequency of competitive interactions at the feed bunk (displacement and attempts to displace) were 250 to $279 \%$ higher during the nonuniform TMR baseline period relative to the days heifers were offered a uniformly distributed TMR-H, TMR-M, or TMR-L diet $(P \leq 0.003 ;$ Figure 4B).

During the nonuniform baseline period, the average FSI $( \pm$ SE) was $192 \pm 42$ s. Relative to this baseline, heifers spent longer at a feeding station when offered uniform TMR-H (average FSI $=335 \pm 42 \mathrm{~s} ; P$ $=0.009)$, tended to have a shorter FSI when offered uniform TMR-L $(102 \pm 42 \mathrm{~s} ; P=0.09)$, and had a
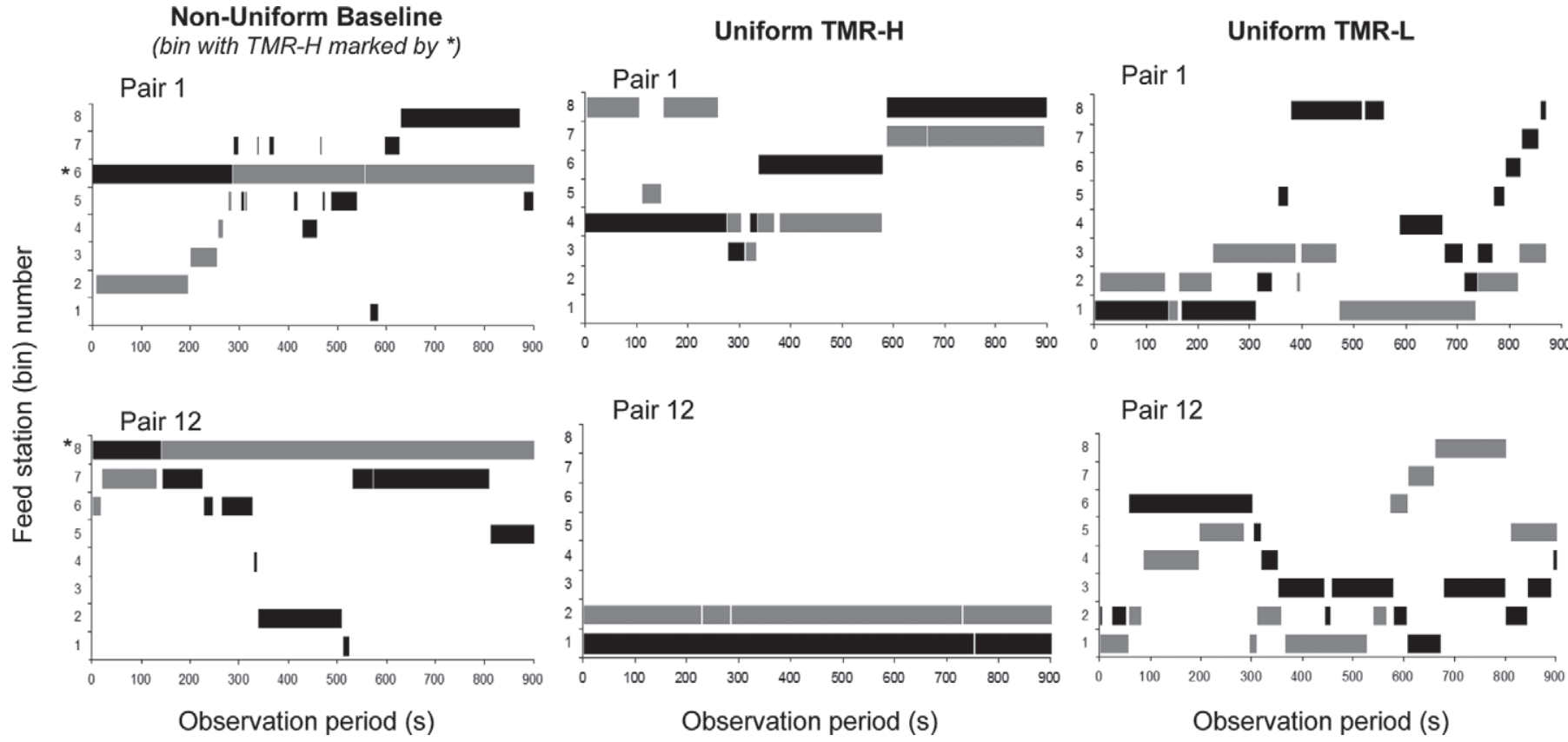

Figure 2. Examples of switching behavior for 2 pairs of heifers observed during the 15-min (900-s) observation period of trial 2. Data are from pair no. 1 and no. 12 (black and gray bars represent the 2 heifers in each pair) during 1 nonuniform baseline TMR day [low-energy TMR (TMR-L) in 7 feed stations and high-energy TMR (TMR-H) in 1 feed station] and during the uniform TMR-H and TMR-L test days. 

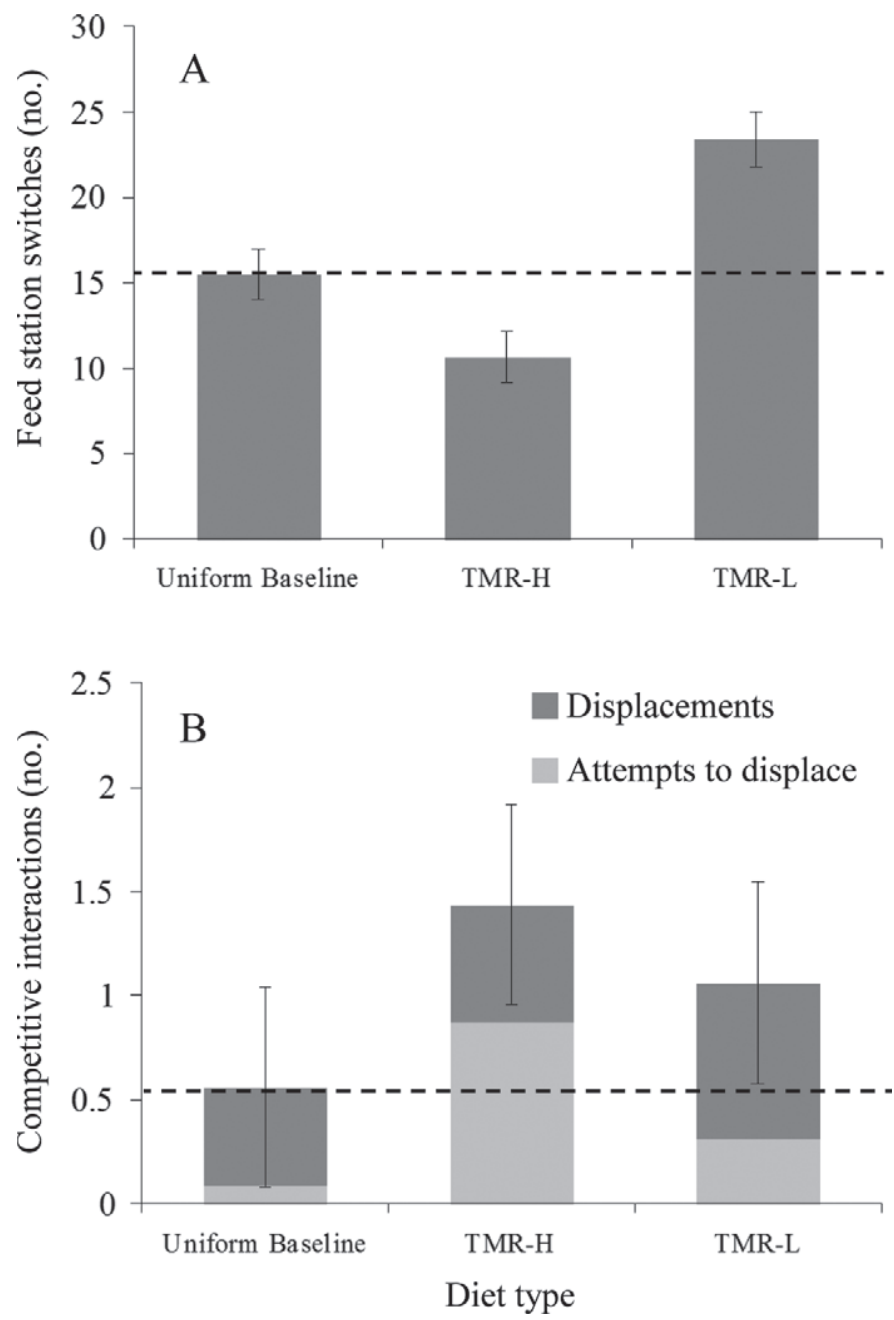

Figure 3. Frequency of feed station switches (A; no. per 15-min observation period) and competitive interactions (B; no. per 15-min observation period) of heifers fed a uniform baseline TMR [moderateenergy TMR (TMR-M) in all 8 feed stations] relative to the 2 test diets [low-energy TMR (TMR-L) or high-energy TMR (TMR-H) in all 8 feed stations].

similar FSI when fed uniform TMR-M $(244 \pm 42 \mathrm{~s} ; P=$ 0.33). Heifers within a pair differed in their average FSI as evidenced by FSI ratios greater than 1; this asymmetry was greatest during the nonuniform baseline period where the FSI ratio [2.7 (95\% CI: 2.1 to 3.6)] was greater than during all other test days [TMR-H: 1.8 (1.4 to 2.4 ), $P=0.04$; TMR-M: 1.9 (1.4 to 2.5$), P$ $=0.07$; and TMR-L: 1.8 (1.4 to 2.4), $P=0.04]$. During the nonuniform baseline period, the heifer within a pair that was most successful at maintaining access to the high-quality bin spent, on average, $632 \pm 38$ s feeding at this location, whereas its partner averaged $136 \pm 31$ $\mathrm{s}$ at the high-quality bin $(P<0.001)$.

\section{DISCUSSION}

Cattle are information gatherers and they use this information to make decisions on when, where, what, and how to eat (Bailey, 1996). One way that this information can be obtained is by direct personal interactions with the environment (Dall et al., 2005). The results from the current study provide the first evidence that indoor-housed cattle engage in exploratory behavior that appears to provide them with information about the quality of feed available. Decisions to either remain at the current feeding location, or move to a new site, appeared to be based on this assessment relative to expectations based on prior experience. Feed station switching increased when heifers were provided a diet that was lower in energy density than that experienced during the baseline period; these changes in behavior likely reflected the expectation that higher-quality feed was available at another station. Similarly, the reduction in switching when cattle were offered a diet of higher energy density than previously experienced suggested that the animals now judged that little chance existed of finding better feed at another location.

Research with grazing yearling steers has also shown that ruminants have the capacity to remember spatial locations that are nutrient rich or that have productive plant communities and will preferentially select these sites when given the opportunity and avoid sites that they have already foraged (Bailey et al., 1989). In freeroaming grazing cattle, preferences for plant communities were found to be proportional to the relative differences in quality (preference for higher CP percentage) and quantity of preferred forage (Senft et al., 1985). Research conducted with other species has also shown that animals will track temporal and spatial changes in site quality by environmental sampling. Chipmunks, for example, increase exploration time when seed collection rate is low due to low patch seed density (Kramer and Weary, 1991). A series of studies have found that starlings, pigeons, and hummingbirds will preferentially select a stable moderate-quality site when an alternative site that varies in quality is in its poor phase, but these animals will switch to the variable site after exploratory sampling has confirmed that quality at the alternate site is higher than the stable site (Tamm 1987; Shettleworth et al., 1988; Inman 1990). In those studies and in the present study, the selection of feed sites and movements between sites appeared to be based on feed decisions aimed at maximizing net energy intake per unit of time, the central concept in optimal foraging theory (Schoener, 1971; Charnov 1976).

Uncertainty about the state or quality of an alternate feeding site is what necessitates the need to sample. In 

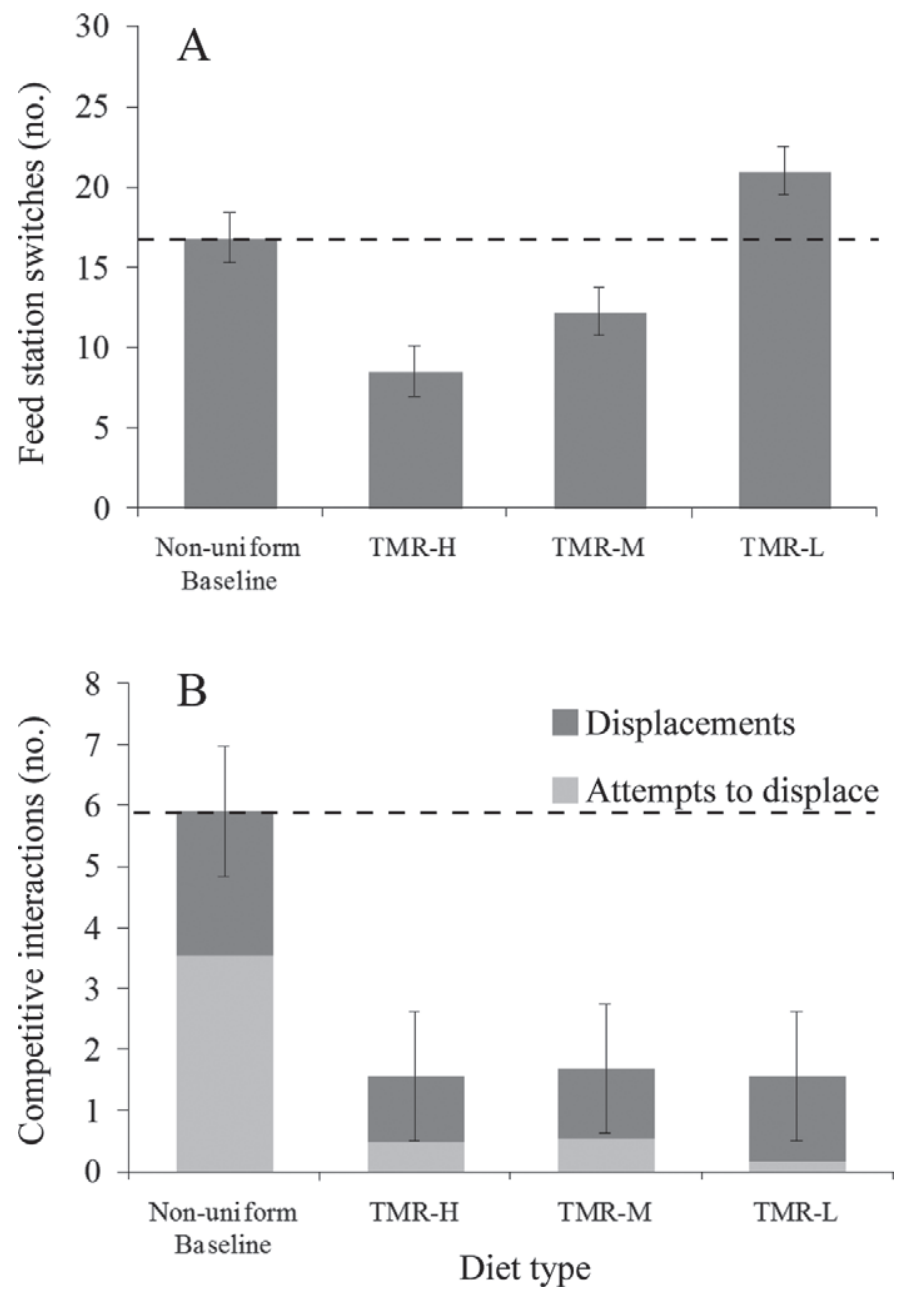

Figure 4. Frequency of feed station switches (A; no. per 15-min observation period) and competitive interactions (B; no. per 15-min observation period) of heifers fed a nonuniform baseline TMR [low-energy TMR (TMR-L) in 7 feed stations and high-energy TMR (TMR-H) in 1 feed station] relative to 3 uniform diets [TMR-L, moderate-energy TMR (TMR-M), or TMR-H in all 8 feed stations].

the studies described above, the more information that animals had about the quality of the variable feeding site (i.e., whether it was in a good or bad state), the better it was able to modify its sampling behavior to maximize intake (Stephens, 1987). In modern dairy production systems, changes in feed quality may be both predicable and unpredictable. Decreasing feed quality at the feed bunk due to sorting behavior (DeVries et al., 2005) may result in increased variation in diet quality over the course of a day; in such cases, we predict that cows will increase sampling to locate less sorted feed sites at later times of the day. In the present study, spatial and temporal changes in feed quality were not predictable and, thus, cattle had to rely on prior dietary experience and sampling to inform feeding decisions.
Diet selection through environmental sampling will be influenced by sensory cues (i.e., taste, texture, and smell, among others) and postingestive feedback (i.e., satiety or malaise resulting from toxicosis) as well as the interaction between these 2 mechanisms. Depending on the digestibility of the diet, dietary feedback mechanism in ruminants can occur within 15 to 60 min following initial feed ingestion (Provenza, 1995); therefore, in the present study, where behavior was only observed for 15 min following initial access to fresh feed, feed site selection and sampling behavior was more likely influenced by sensory cues (e.g., palatability) than postingestive feedback. Over time, heifers may have adjusted their sampling behavior in accordance to postingestive feedback. For example, research has shown that although ruminants prefer high-energy foods such as grain, overconsumption of grain is associated with postingestive malaise and reduced intake likely due to a buildup of byproducts from microbial fermentation (Provenza et al., 1994; Owens et al., 1998); ruminants will subsequently limit intake of grain and chose alternative foods if grain is overconsumed (Phy and Provenza, 1998). Consequently, more exploratory behavior may have been observed over time by heifers transitioned to the TMR-H diet if postingestive mechanisms increased their motivation to find less energy-dense feeding sites. These adaptations are likely important for maintaining nutritional health and future work should explore the long-term effects of changes in dietary energy density on the sampling behavior of cattle raised in indoor production systems.

Clearly, the ability of cattle to effectively adapt to variability in dietary energy density would be impaired if changes were unexpected and frequent as with poor feed bunk management. Given the evidence from the current study that the sampling behavior of heifers is contingent upon their previous experience, we conclude that consistency in feed quality along the feed bunk and across days is important to reduce exploratory sampling.

When an animal uses memory of a previously experienced reward to make a decision about the value of the next reward, the corresponding behavioral change can be considered a "successive contrast" (Flaherty, 1996); the magnitude of the behavioral contrast effect can depend on the degree of difference between the 2 rewards experienced (DiLollo and Beez, 1966). In trial 2 , evidence existed that cattle were sensitive to the magnitude of shift in feed reward between days; a $49 \%$ decrease in feeding station switches occurred when cattle where shifted to the TMR-H diet, but only a $27 \%$ decrease in feeding station switches when shifted to the TMR-M diet; TMR-M was higher in energy than 
the nonuniform baseline diet of trial 2, but lower than TMR-H. These results demonstrate that behavioral responses to changes in diet quality will be influenced by the magnitude of change in diet quality experienced; the greater the change, the greater the behavioral response. These observations may help to explain acute changes in feeding behavior of dairy cattle in response to routine diet changes such as when cattle are transitioned to a lower-energy diet to facilitate the cessation of milk production at dry off.

The study of dietary contrasts may also have application for future studies on dairy cattle welfare. One novel application of the successive contrast paradigm has been for the study of animal emotion (Burman et al., 2008). Increased sensitivity to reward loss appears to be symptomatic of negative affective states in humans. Changes in the magnitude of behavioral responses to shifts in feed rewards may be useful as a method of assessing affective states in cattle.

In the present study, not only did cattle change their sampling frequency when exposed to changes in the quality of TMR at the feed bunk, they also changed the time spent at each feed station. In general, when dietary energy was lower than previously experienced, FSI decreased. Ruyle and Dwyer (1985) observed a similar response in grazing sheep; as patch quality decreased due to diminished forage supply, grazing sheep responded by increasing the number of FSI that were of short duration $(<20 \mathrm{~s})$ and moved between patches more frequently.

Social factors also likely played an important role in the sampling behavior of heifers in the current study. Social animals, including cows and sheep, are motivated to remain close to one another (Scott et al., 1995; Dumont and Boissy, 2000) and, while foraging, are more likely to join a patch where other individuals are feeding rather than an alternate unoccupied patch (Pöysä, 1992). In the present study, it appeared that at times, the movements of heifers within a pair paralleled each other. This behavior was particularly evident when the heifers were shifted to the TMR-H diet in trial 2 (see Figure 2).

Social cues may have signaled the presence of a superior feeding site during nonuniform baseline period. Inman (1990) studied the social foraging behavior of starlings exposed to patches with different densities of buried mealworms and found that that although the birds tended to avoid patches that already contained a large number of individuals, they were attracted to sites containing a high proportion of foraging birds that had already discovered food. Therefore, it may not be merely the presence of individuals at a site that attracts other foragers, but rather subtle behavioral cues that signal the presence of a superior food source. More research is required to identify whether specific behavioral cues exist that alert other cattle to the presence of a superior feeding location at the feed bunk.

If social cues motivate cattle to sample a new feed site, it will still likely be the information gained from the direct personal interaction with the environment that determines whether the individual chooses to stay or move to another feed site. The results of this study suggest that cattle can quickly assess whether TMR quality is spatially uniform and will respond to this consistency by engaging in fewer competitive interactions even when the average quality is lower than previously experienced (i.e., exposure to TMR-L after nonuniform baseline treatment).

During the nonuniform baseline treatment, only one heifer could eat at the high quality feed station at a time and the other heifer was forced to either wait for access to the high-quality station or eat a lower-energy diet. The higher FSI ratio during the nonuniform baseline period in trial 2 confirms this asymmetry; during the nonuniform baseline period, one cow in a pair had a longer average FSI than her partner; during the experimental test days (when feed distribution was uniform), FSI were more similar within pairs. This is further evidence that variable feeding environments contribute to inefficient feeding behavior. Spatial variation in feed quality will ultimately result in within-group variation in the quality of feed consumed, potentially affecting health and performance measures. Future work in this area should explore how sampling behavior is affected when more than 2 cows are present at the feed bunk, as increased competition for feed may increase the amount of within-group variation in the quality of feed consumed. Increased stocking density at the feed bunk is known to reduce feeding time, increase feeding rate, and competitive interactions, with socially subordinate animals being most affected (Huzzey et al., 2006; Proudfoot et al., 2009).

Some have argued that the application of optimal foraging theory to large grazing herbivores such as cattle is inappropriate because unlike predators or nectar feeders that seek spatially scattered prey of high quality, large grazing herbivores must contend with lower-quality and more evenly distributed feed sources (Owen-Smith and Novellie, 1981; Senft et al., 1987). The results of the current study suggest that this critique is misguided; modern dairy farms intentionally and unintentionally vary diet quality in time and space with potentially important effects on cattle foraging behavior; we urge new research aimed at expanding our understanding of the foraging behavior of cattle reared in these systems. 


\section{CONCLUSIONS}

Dairy heifers sample feed quality by changing feeding locations at the feed bunk; this sampling behavior is affected by variability in energy density of the diet and the contrast between the current dietary experience and the previous experience. In general, exposure to a diet that is lower in quality to that previously experienced elicits a higher frequency of switches between feed stations and a shorter average time spent at each feed station (shorter FSI); exposure to a TMR that is higher in quality than previously experienced results in fewer switches and longer FSI. Understanding how animals react to unexpected changes in reward (e.g., diet quality) is important because it provides insight into the strategies cattle use to learn about their environment. A key finding of this study was that competitive interactions increase when TMR quality is nonuniform across the feed bunk, reflecting the motivation of individual heifers for obtaining access to the higher-quality feed. These results indicate that consistency in feed bunk management will help reduce competitive interactions, minimize time cattle spend grazing the feed bunk, and ultimately help ensure that the animals in a group have similar opportunities to obtain access to the intended diet.

\section{ACKNOWLEDGMENTS}

We thank Don Kramer (McGill University, Montreal, QC, Canada) for discussions about sampling behavior that helped in the interpretation of the results presented here. We are also grateful to Su Jung Lim (Wageningen University, Wageningen, the Netherlands), whose undergraduate thesis work provided the initial inspiration for the current experiment. We also thank the students, staff, and especially Nelson Dinn, of the University of British Columbia Dairy Education and Research Centre (Agassiz, BC, Canada) for their help throughout the study. This study was funded by a Discovery Grant from the Natural Sciences and Engineering Research Council (NSERC; Ottawa, ON, Canada). The University of British Columbia's Animal Welfare Program is also funded by NSERC's Industrial Research Chair Program with industry contributions from the Dairy Farmers of Canada (Ottawa, ON, Canada), Westgen Endowment Fund (Milner, BC, Canada), Pfizer Animal Health (Kirkland, QC, Canada), BC Cattle Industry Development Fund (Kamloops, BC, Canada), the BC Milk Producers (Burnaby, BC, Canada), BC Dairy Foundation (Burnaby, BC, Canada), BC Dairy Industry Research and Education Fund (Abbotsford, BC, Canada), and Alberta Milk (Edmonton, AB, Canada).

\section{REFERENCES}

Bailey, D. W. 1996. Mechanisms that result in large herbivore grazing distribution patterns. J. Range Manage. 49:386-400.

Bailey, D. W., L. R. Rittenhouse, R. H. Hart, D. M. Swift, and R. W. Richards. 1989. Association of relative food availabilities and locations by cattle. J. Range Manage. 42:480-482.

Burman, O. H. P., R. M. A. Parker, E. S. Paul, and M. Mendl. 2008. Sensitivity to reward loss as an indicator of animal welfare and emotion. Biol. Lett. 4:330-333.

CCAC (Canadian Council on Animal Care). 2009. CCAC guidelines on: The care and use of farm animals in research, teaching and testing. Canadian Council on Animal Care, Ottawa, ON, Canada.

Charnov, E. L. 1976. Optimal foraging, the marginal value theorem. Theor. Popul. Biol. 9:129-136.

Dall, S. R. X., L.-A. Giraldeau, O. Olsson, J. M. McNamara, and D. W. Stephens. 2005. Information and its use by animals in evolutionary ecology. Trends Ecol. Evol. 20:187-193.

DeVries, T. J., and M. A. G. von Keyserlingk. 2005. Time of feed delivery affects the feeding and lying patterns of dairy cows. J. Dairy Sci. 88:625-631.

DeVries, T. J., M. A. G. von Keyserlingk, and K. A. Beauchemin. 2005. Frequency of feed delivery affects the behavior of lactating dairy cows. J. Dairy Sci. 88:3553-3562.

DiLollo, F. D., and V. Beez. 1966. Negative contrast effect as a function of magnitude of reward decrement. Psychon. Sci. 5:99-100.

Dumont, B., and A. Boissy. 2000. Grazing behavior of sheep in a situation of conflict between feeding and social motivations. Behav. Processes 49:131-138.

Flaherty, C. F. 1996. Incentive Relativity: Problems in the Behavioral Sciences. Cambridge University Press, New York, NY.

Huzzey, J. M., T. J. DeVries, P. Valois, and M. A. G. von Keyserlingk. 2006. Stocking density and feed barrier design affect the feeding and social behavior of dairy cattle. J. Dairy Sci. 89:126-133.

Inman, A. J. 1990. Foraging decisions: The effects of conspecifics and environmental stochasticity. PhD Diss. University of Oxford, Oxford, UK.

Kramer, D. L., and D. M. Weary. 1991. Exploration versus exploitation: A field study of time allocation to environmental tracking by foraging chipmunks. Anim. Behav. 41:443-449.

Leonardi, C., and L. E. Armentano. 2003. Effect of quantity, quality, and length of alfalfa hay on selective consumption by dairy cows. J. Dairy Sci. 86:557-564.

Lima, S. L. 1998. Stress and decision making under the risk of predation: Recent developments from behavioral, reproductive, and ecological perspectives. Adv. Stud. Behav. 27:215-290.

Olofsson, J. 1999. Competition for total mixed diets fed for ad libitum intake using one or four cows per feeding station. J. Dairy Sci. 82:69-79.

Owen-Smith, N., and P. Novellie. 1981. What should a clever ungulate eat? Am. Nat. 119:151-178.

Owens, F. N., D. S. Secrist, W. J. Hill, and D. R. Gill. 1998. Acidosis in cattle: A review. J. Anim. Sci. 76:275-286.

Phy, T. S., and F. D. Provenza. 1998. Sheep fed grain prefer foods and solutions that attenuate acidosis. J. Anim. Sci. 76:954-960.

Pöysä, H. 1992. Group foraging in patchy environments: The importance of coarse-level local enhancement. Ornis Scand. 23:159-166.

Proudfoot, K. L., D. M. Veira, D. M. Weary, and M. A. G. von Keyserlingk. 2009. Competition at the feed bunk changes the feeding, standing, and social behavior of transition dairy cows. J. Dairy Sci. 92:3116-3123.

Provenza, F. D. 1995. Postingestive feedback as an elementary determinant of food selection and intake in ruminants. J. Range Manage. 48:2-17.

Provenza, F. D., L. Ortega-Reyes, C. B. Scott, J. J. Lynch, and E. A. Burritt. 1994. Antiemetic drugs attenuate food aversions in sheep. J. Anim. Sci. 72:1989-1994.

Ruyle, G. B., and D. D. Dwyer. 1985. Feeding stations of sheep as an indicator of diminished forage supply. J. Anim. Sci. 61:349-353.

Schoener, T. W. 1971. Theory of feeding strategies. Annu. Rev. Ecol. Syst. 2:369-404. 
Scott, C. B., F. D. Provenza, and R. E. Banner. 1995. Dietary habits and social interactions affect choice of feeding location by sheep. Appl. Anim. Behav. Sci. 45:225-237.

Senft, R. L., M. B. Coughenour, D. W. Bailey, L. R. Rittenhouse, O. E. Sala, and D. M. Swift. 1987. Large herbivore foraging and ecological hierarchies. Bioscience 37:789-795,798-799.

Senft, R. L., L. R. Rittenhouse, and R. G. Woodmansee. 1985. Factors influencing patterns of cattle grazing behavior on shortgrass steppe. J. Range Manage. 38:82-87.
Shettleworth, S. J., J. R. Krebs, D. W. Stephens, and J. Gibbon. 1988 Tracking a fluctuating environment: A study of sampling. Anim. Behav. 36:87-105.

Stephens, D. W. 1987. On economically tracking a variable environment. Theor. Popul. Biol. 32:15-25.

Stephens, D. W., J. S. Brown, and R. C. Ydenberg. 2007. Foraging Behavior and Ecology. The University of Chicago Press, Chicago, IL

Tamm, S. 1987. Tracking varying environments: Sampling by hummingbirds. Anim. Behav. 35:1725-1734 